\title{
Propriedades das Séries Temporais dos Lucros Trimestrais das Empresas Brasileiras Negociadas em Bolsa
}

\author{
Thiago Rocha Fabris* \\ Newton Carneiro Affonso da Costa Jr.**
}

\begin{abstract}
Resumo
Este artigo analisa o comportamento das séries temporais de lucros trimestrais (operacional e líquido) de uma amostra de 109 empresas brasileiras com ações negociadas em bolsa, durante o período de 1995 a 2008. Foram utilizados cinco modelos de previsão de séries de lucros já previamente estimados e consagrados na literatura internacional, denominados aqui de modelos predeterminados, bem como foi empregado o método de Box e Jenkins (BJ) para verificar a possibilidade de se encontrar um modelo mais adequado às condições do mercado brasileiro. Verifica-se que somente o lucro operacional é passível de previsão, não sendo possível a aplicabilidade do método de BJ ao lucro líquido, pois este apresenta, na amostra estudada, um comportamento aleatório, descrito por um random walk com deslocamento. Conclui-se, também, que não existe um modelo ARIMA predeterminado que seja aplicável ao conjunto das empresas estudadas. Um modelo deve ser identificado individualmente para cada empresa.
\end{abstract}

Palavras-chave: previsão de lucros; lucros trimestrais; modelos ARIMA.

Códigos JEL: G17; M21.

\section{Abstract}

This paper analyzes the time series behavior of quarterly earnings (operating and net) of a sample of 109 Brazilian listed companies during the period 1995 to 2008. To perform the study, we used five models of earnings forecasting that were previously estimated in the international literature, here called common-structure models, as well as using the Box end Jenkins (BJ) method to verify the possibility of finding a model appropriate to the conditions of the Brazilian market. Empirical evidence is provided showing that only operating earnings can be predicted. It is not possible to apply BJ method to predict net income, as it presents a random behavior, described by a random walk with drift. Also, it is not possible to identify any common-structure ARIMA model for all companies. A model should be identified individually for each company.

Keywords: earnings forecasting; quarterly earnings; ARIMA models.

\footnotetext{
Submetido em Fevereiro de 2010. Aceito em Março de 2010. O artigo foi avaliado segundo o processo de duplo anonimato além se de ser avaliado pelo editor. Editor responsável: Ricardo P. C. Leal.

*Universidade do Extremo Sul Catarinense - UNESC, Santa Catarina, Brasil.

E-mail: thiagorfabris@unesc.net

**Universidade Federal de Santa Catarina - UFSC, Santa Caratina, Brasil.

E-mail: ncacjr@gmail.com
} 


\section{Introdução}

As propriedades das séries temporais de lucros e de outras séries financeiras têm sido amplamente estudadas na literatura internacional desde os anos 1960. Do ponto de vista teórico, de acordo com Watts e Zimmerman (1986), são três os fatores que têm motivado o desenvolvimento desta literatura. O primeiro deles foi a necessidade de se desenvolver melhores modelos para a avaliação de títulos, principalmente ações. Os pesquisadores buscavam melhores previsões de lucros para o cálculo de fluxos de caixa futuros. A segunda motivação derivou da busca por um melhor modelo de expectativas de lucros por parte dos pesquisadores que estudavam o relacionamento entre preços de ações e lucros contábeis, como no trabalho seminal de Ball e Brown (1968). A terceira motivação relaciona-se à tentativa de explicar a escolha, por parte da administração da empresa, de determinados procedimentos contábeis. A literatura sobre suavização de lucros (income smoothing) está associada a essa última motivação.

Do ponto de vista gerencial, várias partes estão interessadas no comportamento temporal das séries financeiras. Foster (1986), por exemplo, cita três partes interessadas: (1) analistas financeiros que costumam apresentar em seus relatórios as previsões de lucros e de outras variáveis relevantes das empresas sob análise; (2) instituições provedoras de recursos que costumam incluir em seus procedimentos de concessão de crédito alguma previsão dos lucros e fluxos de caixa de seus clientes durante o período do empréstimo; (3) a administração interna da empresa que na atividade de estabelecer estratégias corporativas procura estimar os lucros e fluxos de caixa futuros a partir de diferentes composições de sua estrutura de capitais e de diferentes decisões de investimentos.

Subjacente a todas as motivações e fatores acima mencionados está a necessidade de se conhecer os lucros futuros, ou seja, a previsão de lucros das empresas. Esta previsão poderá ser feita, segundo Foster (1986), mecanicamente, através de modelos econométricos, ou através de previsões de analistas. O presente artigo tem como foco o uso de modelos econométricos univariados. ${ }^{1}$

Os primeiros trabalhos empíricos sobre as propriedades das séries temporais de lucro concluíram que as variações nos lucros (anuais) de empresas britânicas e norte americanas eram imprevisíveis, ou seja, seguiam um random walk. ${ }^{2}$

\footnotetext{
${ }^{1} \mathrm{O}$ leitor interessado na comparação de previsões de modelos mecânicos com aquelas feitas por analistas, pode consultar os trabalhos de Brown e Rozeff (1978), Collins e Hopwood (1980) e Brown et alii (1987) para o mercado norte americano e Ness Jr. (1995) e Silva (1998) para o Brasil. Em geral, verifica-se que analistas têm vantagem, nem sempre significativa, nas previsões de curto prazo, pois podem incorporar mais informações em suas análises. Em contrapartida são mais caros para as empresas. Nas previsões de longo prazo, que são usadas em modelos de avaliação e nos cálculos de custo de capital, as vantagens são mais difusas.

${ }^{2}$ De acordo com Freeman et alii (1982), esta denominação não é muito precisa, pois, na verdade, o consenso é de que os lucros (anuais) seguem processos denominados de martingale ou de submartingale. Assim, uma série temporal descrita por $X_{t}=X_{t-1}+\delta+e_{t}$ se caracteriza por um processo do tipo martingale se o drift for igual a zero $(\delta=0)$ e a média da distribuição dos erros for zero $\left(E\left(e_{t}\right)=0\right)$. Um processo do tipo submartingale, por outro lado, pode ser descrito pela relação anterior, mas $\operatorname{com} \delta \geq 0$. Se, adicionalmente, a série dos erros $\left(e_{t}, e_{t+1}, e_{t+2}, \ldots, e_{t+n}\right)$ for independente
} 
Ball e Watts (1972) publicaram um dos primeiros estudos sobre o comportamento temporal de séries financeiras e mostraram que, em média, o lucro anual e o lucro por ação podem ser descritos por um processo submartigale. Esta constatação experimental é uma das mais robustas na literatura pertinente. Watts e Leftwich (1977) utilizaram o método de Box e Jenkins (1976) para analisar os lucros anuais de diversas empresas e concluíram que as séries temporais analisadas também seguem um submartingale. Diversos trabalhos corroboram que a melhor previsão para os lucros anuais seria descrito por seu valor no período anterior mais um choque aleatório. Destacam-se nesta vertente os estudos de Little (1962), Ball e Watts (1979), Whittred (1978), Kinnunen (1988, 1991), Bao e Bao (1996), Hamman et alii (2002) e Chan et alii (2003).

Resultados opostos foram encontrados por Beaver (1970), Brooks e Buckmaster (1976) e Freeman et alii (1982). Estes autores apontam para a não-ocorrência de random walk para os lucros anuais das empresas e atestaram, inclusive, a existência de uma tendência de reversão à média nas variações de lucros, caracterizando um processo estacionário. Esses estudos concluem favoravelmente pela utilização das séries históricas de lucro para um horizonte infinito de avaliação, isto é, assumem, em certo sentido, uma possível previsibilidade dos lucros (anuais) das empresas. Outros trabalhos mais recentes nesta vertente são os de Wu et alii (1996), Khorana et alii (1999), Fama e French (2000) e Harris e Marston (2001), para as empresas norte americanas.

No que concerne aos lucros trimestrais destaca-se, inicialmente, o trabalho de Brown e Neiderhoffer (1968), que mostraram a utilidade dos lucros trimestrais nas previsões dos lucros anuais. O interesse pelos lucros trimestrais é justificado em função das características sazonais de muitos setores industriais. Estes ainda refletem de modo mais acurado as oscilações do mercado se comparado às previsões de lucros anuais (Kothari, 2001).

Posteriormente, Reilly et alii (1972) corroboram em favor dos dados trimestrais se comparados aos dados anuais quando usados em previsões de séries temporais financeiras. Brown e Rozeff (1979), Foster (1977) e Griffin (1977) utilizaram a metodologia de Box e Jenkins para demonstrar que as séries analisadas podem ser descritas pelos modelos ARIMA (100) x (011), ARIMA (100) x (010) e ARIMA (011) x (011), respectivamente. ${ }^{3}$ Martinez et alii (2008), que analisam a presença de independência estatística entre as variações sucessivas de lucro trimestral de empresas brasileiras, destacam que se as mudanças nos lucros apresentam um

\footnotetext{
e identicamente distribuída, o processo é denominado random walk.

${ }^{3}$ Num modelo ARIMA $(p, d, q) \times(P, D, Q)_{S}$, para uma série temporal $Y, p$ refere-se à ordem da dimensão autorregressiva (AR) do modelo, $d$ refere-se ao número de vezes que a série deve ser submetida a diferenças para torná-la estacionária e $q$ é o número de valores defasados do termo de erro da série, representando a ordem da dimensão média móvel (MA) do modelo. Quando os dados são observados em períodos inferiores a um ano, por exemplo $s=4$ para dados trimestrais, a série pode apresentar autocorrelação em períodos de sazonalidades $(P)$, pode ser necessário diferenciações sazonais $(D)$ e pode apresentar média móvel sazonal $(Q)$. Maiores detalhes sobre a modelagem ARIMA são apresentados adiante, na seção 3.2 deste artigo.
} 
comportamento temporal do tipo random walk este fato apresenta uma série de implicações para investidores e demais stakeholders da empresa. Uma delas é que se a premissa for válida, as mudanças nos lucros futuros de empresas não podem ser previstas a partir da série passada.

Sendo assim, o presente artigo tem por finalidade analisar o comportamento das séries trimestrais de lucro líquido e lucro operacional de empresas brasileiras de capital aberto no período de 1995 a 2008. Para tanto, serão utilizados alguns dos modelos já previamente estimados e consagrados na literatura internacional, bem como será empregado o método de Box e Jenkins (1976) para verificar se é possível encontrar um modelo mais adequado às condições do mercado brasileiro.

Esta pesquisa é relevante pois uma estimativa mais precisa de lucros futuros é essencial para diversos campos de estudos em finanças: (1) avaliação de empresas; (2) determinação do custo de capital; (3) estudos de evento - estabelecimento de uma relação precisa entre lucros não antecipados e retornos das ações; (4) estudos recentes procuram melhor esclarecer a relação entre projeções de crescimento de lucros, e outras variáveis financeiras, com previsões para o equity risk premium (Chan et alii, 2003, Fama e French, 2002); (5) a regulamentação contábil norte americana e internacional está cada vez mais exigindo informações mais precisas de lucros (cash flow e accruals) e suas previsões (Lorek e Willinger, 2008, Dechow e Dichev, 2002, Lobo et alii, 1998).

No âmbito gerencial, o desenvolvimento de modelos econométricos adaptados às características peculiares a cada setor econômico possibilita à administração da empresa estabelecer estratégias de monitoramento mais precisas e, também, que analistas possam obter melhores previsões das séries financeiras.

Além do mais, apesar de ser um tema bastante debatido a nível internacional, publicações em âmbito nacional que aplicam a metodologia ARIMA para estudar o comportamento das séries de lucros das empresas brasileiras é praticamente inexistente. $^{4}$

Na próxima seção apresenta-se, de forma sucinta, alguns dos principais trabalhos sobre o estudo do comportamento temporal de séries financeiras. A terceira seção apresenta a metodologia aplicada aos dados e a metodologia proposta por Box e Jenkins (1976). A quarta seção detalha os resultados facilitando a visualização dos testes utilizados para sustentar as conclusões. A última seção apresenta as conclusões sobre o tema.

\footnotetext{
${ }^{4}$ Exceção deve ser feita às dissertações de mestrado, não publicadas, de Silva (2000) e Cunha (2004) na PUC-Rio. Aplicando a metodologia ARIMA para séries financeiras trimestrais de empresas negociadas na BOVESPA, Silva (2000) verificou somente ser possível prever a receita líquida, não sendo possível aplicar essa mesma metodologia às séries históricas de lucro operacional e líquido. Cunha (2004), por sua vez, mostra que as taxas de crescimento das séries anuais de receita bruta, EBITDA e lucro operacional podem ser descritas apenas por um processo random walk ou random walk com drift (deslocamento).
} 


\section{Revisão da Literatura}

O foco sobre os lucros (anuais) das empresas remonta à década de 1960, com os trabalhos de Little (1962) e de Little e Rayner (1966). Ambos analisaram o comportamento das taxas de crescimento dos lucros de empresas britânicas. Os autores apontaram pela aleatoriedade das séries, fenômeno este que ficou conhecido como Higgledy Piggledy Growth.

Ball e Watts (1972), Albrecht et alii (1977), Watts e Leftwich (1977) observam que os modelos ARIMA não geram melhores previsões se comparados com um modelo do tipo random walk para as séries de lucros anuais das empresas norte americanas.

Watts e Leftwich (1977) também indicaram que os lucros anuais das empresas têm um comportamento descrito por um modelo submartingale. Também verificaram, em algumas séries, a existência do modelo martingale.

Adicionalmente, o estudo de Bao e Bao (1996) para empresas tailandesas aponta, entre quatro modelos previamente definidos pelos autores, favoravelmente para o processo de random walk como sendo o mais apropriado para delinear as oscilações dos lucros, isto é, não existem evidências quanto à correlação entre taxas sucessivas de crescimento de lucros. Na mesma linha, situa-se o trabalho de Capstaff et alii (1998), que mostra a maior dificuldade em prever resultados de firmas britânicas com resultados históricos, os autores concluem a favor da nãopersistência das variáveis defasadas.

Encontram-se ainda vários outros estudos sobre os lucros anuais em diversos países, também mostrando que o processo random walk apresenta os menores erros de previsão para estas séries, como na Nova Zelândia com Caird e Emanuel (1981), Firth (1982) e Austin e Graydon (1993). Na Austrália com Finn e Whittred (2007) e Taylor e Tress (1988). Em Ariff e Aw (1987) mostraram que os lucros pareciam mudar randomicamente para as empresas de Singapura. No Brasil, Cunha (2004) mostra que as séries temporais anuais da receita bruta, EBITDA e lucro operacional, de uma amostra de empresas negociadas na BOVESPA, podem ser descritas apenas por um processo random walk ou random walk com drift (deslocamento).

Embora fosse comum acreditar até os anos 70 que os lucros anuais tivessem um comportamento random walk ou random walk com drift, alguns pesquisadores argumentavam que os lucros anuais eram mais bem descritos por modelos de reversão à média (Beaver, 1970, Brooks e Buckmaster, 1976, Salamon e Smith, 1977, Beaver e Morse, 1978, Freeman et alii, 1982). Os modelos de reversão à média conhecidos como ARIMA (011) ou IMA (011), têm sido defendidos por diversos pesquisadores tais como: Beaver et alii $(1980,1987)$, Collins e Kothari (1989), Kendall e Zarowin (1990) e Ramakrishnan e Thomas (1992). 
No que concerne aos lucros trimestrais, podemos destacar estudos clássicos que serviram de base para outros estudos: Watts (1975), Griffin (1977), Foster (1977) e Brown e Rozeff (1979). As evidências empíricas demonstram que os lucros trimestrais podem ser modelados de acordo com a metodologia Box e Jenkins, contrariando as evidências encontradas dos lucros anuais (random walk ou martingale).

Griffin (1977) examinou o comportamento dos lucros trimestrais e as relações entre lucros e ações. A metodologia de Box e Jenkins foi utilizada para identificar os modelos (ARIMA) mais apropriados. A amostra foi composta pelas 94 maiores companhias listadas na bolsa de valores de Nova York. O trabalho concluiu que os lucros trimestrais podem ser modelados por um processo ARIMA (011) x (011), isto é, um componente de média móvel regular e sazonal, ambos estacionários em primeira diferença. O estudo procurou mostrar que os lucros trimestrais não seguem um processo random walk ou um martingale. Basicamente, quatro modelos ARIMA foram testados: um random walk não estacionário - ARIMA (100), média móvel de primeira ordem - ARIMA (001), autorregressivo em primeira diferença - ARIMA (110) e o modelo proposto por Griffin - ARIMA (011) x (011). Os testes estatísticos demonstraram que o melhor processo que descreve o lucro trimestral é uma combinação de média móvel regular e sazonal, ambos em primeira diferença. Os resultados foram baseados na análise cross-section da função de autocorrelação e função de autocorrelação parcial. O mesmo resultado foi encontrado por Watts (1975).

Outro estudo pioneiro na aplicação da metodologia de Box e Jenkins para os lucros trimestrais é o trabalho desenvolvido por Foster (1977). Foi investigado o comportamento de três séries contábeis trimestrais: lucros, vendas e gastos. Sua amostra foi composta por 69 companhias ao longo de 28 anos. Os resultados também foram baseados na função de autocorrelação cross-section. O autor utilizou os primeiros 15 anos para estimar os modelos de cada companhia e os próximos 13 anos foram utilizados para a previsão. A média de todas as observações foram utilizadas para a realização dos testes estatísticos. A conclusão do estudo, no que concerne aos lucros trimestrais, foi que o modelo que mais se ajusta aos dados é descrito por um processo autorregressivo não estacionário e por uma diferença sazonal, dado por um processo ARIMA (100) x (010).

Brown e Rozeff (1979) propuseram um novo modelo ARIMA, cujos resultados foram comparados com a equação descrita nos trabalhos de Watts (1975), Foster (1977) e Griffin (1977). A amostra foi composta por 23 empresas de diversos setores econômicos. A conclusão do autor foi que as previsões dos lucros trimestrais são mais bem descritas por um processo ARIMA (100) x (011).

Muitos estudos têm examinado qual o modelo que gera uma previsão mais acurada para os lucros trimestrais. Benston e Watts (1978) evidenciam em favor do modelo de Foster (1977), Lorek (1979) argumenta em favor do modelo Griffin (1977) e Watts (1975), Collins e Hopwood (1980) e Bathke Jr. e Lorek (1984) defendem o modelo proposto por Brown e Rozeff (1979). 
Outro trabalho recente é o desenvolvido por Lorek e Willinger (2007). Os autores analisaram 1.216 empresas norte americanas divididas em três grandes grupos: grupo composto por empresas de alta tecnologia, grupo composto por empresas com alguma regulação (regulated firms) e o terceiro composto por empresas que não pertenciam aos grupos anteriores. Foram analisados cinco diferentes modelos, a saber: Random Walk com drift (RWD), Seasonal Random Walk com drift (SRWD), o modelo Foster - ARIMA (100) x (010) com drift, o modelo de Brown e Rozeff - ARIMA (100) x (011) e o modelo de Griffin-Watts - ARIMA (011) x (011). A conclusão do estudo destacou que as empresas da primeira e terceira amostra obtiveram resultados inferiores, no sentido de menor previsibilidade, quando comparados aos resultados das empresas reguladas.

\section{Metodologia}

\subsection{Tratamento dos dados}

O presente artigo consiste na análise das propriedades estatísticas e na observação ex-post do comportamento das séries temporais de lucro líquido e operacional de empresas brasileiras com ações negociadas na Bolsa de Valores, Mercadorias e Futuros (BM\&FBOVESPA), durante o período de 1995 a 2008.

Para tanto, foram coletadas as séries de lucro líquido e operacional para todas as empresas listadas na Economática ao final de 1995 a2008. A partir deste universo, selecionou-se aquelas empresas que não apresentaram falhas ao longo das séries, num total de 109 empresas, conforme procedimento adotado por Ball e Watts (1972) e Lorek e Willinger (2007). ${ }^{5}$ Em seguida, para retirar possíveis efeitos de aumento/diminuição na variação dos lucros induzidos pela inflação, as séries foram deflacionadas pelo IPCA, com os dados em moeda constante de dezembro de 2008. Este procedimento também foi utilizado por Kinnunen (1988, 1991) e por Martinez et alii (2008).

Também foi empregada a divisão dos setores econômicos de acordo com a classificação da BM\&FBOVESPA. A divisão setorial também foi usada por Lorek e Willinger (2007). Os estudos realizados através de setores ou indústrias específicas têm obtido maior sucesso se comparados a estudos que não levam em consideração o ambiente econômico em que a empresa está inserida.

Com relação aos dados, estes em sua maioria não sofreram nenhuma alteração, como transformação logarítmica, pois algumas empresas apresentaram valores negativos, o que impossibilita tal transformação.

\footnotetext{
${ }^{5}$ Este processo de seleção da amostra pode provocar o que se denomina viés de sobrevivência. A importância deste viés sobre os resultados obtidos não pode ser determinada com base na presente amostra. Segundo Ball e Watts (1979), uma maneira de se conhecer o tamanho deste problema seria realizar o mesmo experimento com diferentes amostras e em diferentes períodos de tempo. Ball e Watts (1979) afirmam que realizaram tais análises e não encontraram diferenças significativas. Isto não foi realizado no presente trabalho.
} 
A partir da composição das séries de cada empresa foi aplicado o método de Box e Jenkins para identificar e estimar os modelos estatísticos de previsão, além da aplicação dos modelos predeterminados, conhecidos na literatura pertinente como "premier models".

\subsection{O método de Box e Jenkins}

O método de Box e Jenkins, também chamado ARIMA (auto-regressivo, integrado e de médias móveis), permite modelar uma série temporal sem precisar levar em conta as relações econômicas que a geraram. Esta modelagem é feita através da determinação do comportamento da correlação entre os valores sucessivos da série temporal em apreço (autocorrelação) e, com base neste comportamento, realizar previsões sobre os valores futuros.

Inicialmente, tem-se o processo ARMA (p, q) (autoregressivo e de média móvel) que é uma generalização dos modelos AR (p) e MA (q), onde p e q referemse, respectivamente, às ordens auto-regressivas e de média móvel do processo. Este modelo pode ser descrito da seguinte forma:

$$
Y_{t}=\sum_{i=1}^{p} \phi_{i} Y_{t-i}+\mu_{t}+\sum_{j=1}^{q} \theta_{j} \mu_{t-j}
$$

onde $Y_{t}=$ série do lucro líquido e operacional;

$Y_{t-i}=$ série do lucro líquido e operacional defasada $i$ períodos;

$\phi_{i}=$ termo autorregressivo;

$\theta=$ termo de média móvel;

$\mu_{t}=$ termo de erro estocástico que segue uma distribuição gaussiana.

Um modelo do tipo ARMA deve ser aplicado apenas em séries estacionárias ou fracamente estacionárias, ou seja, séries que apresentam médias e variâncias estacionárias e autocovariâncias que dependem apenas das defasagens entre os instantes de tempo (Gujarati, 2006). Quando uma série não se apresenta estacionária é preciso torná-la estacionária para se empregar o método de Box e Jenkins. Assim, se uma série temporal tiver que ser diferenciada $d$ vezes para tornar-se estacionária para então aplicar-lhe o modelo ARMA (p, q), diz-se que a série é um ARIMA (p, $\mathrm{d}, \mathrm{q})$.

Quando os dados são observados em períodos inferiores a um ano, a série também pode apresentar autocorrelação em períodos de sazonalidade. Tais modelos são conhecidos na literatura como SARIMA. Este modelo passa a ser composto também pelos componentes sazonais auto-regressivos e de média móvel, representado como SARIMA (p, d, q) (P, D, Q), em que $d$ é a ordem de diferenciação e $D$ é a ordem de diferenciação sazonal. Assim, $p, P, q$ e $Q$ determinam o número de parâmetros necessários ao modelo e d, D e s identificam as observações passadas influentes nos valores a serem previstos. Ficando, de forma generalizada, ARIMA $(p, d, q)(P, D, Q) s$. 
$Y_{t}=\delta+\sum_{i=1}^{p} \phi_{i} \Delta^{d} Y_{i-1}+\sum_{i=1}^{p} \Phi_{i}^{P} \Delta^{D} Y_{i-1}+\mu_{j}+\sum_{j=1}^{q} \theta_{j} \mu_{j-1}+\sum_{j=1}^{q} \Phi_{j}^{Q} \mu_{j-1}+\epsilon_{t}$

onde:

$\Delta^{d}=$ ordem de diferenciação;

$\Delta^{D}=$ ordem de diferenciação sazonal;

$\Phi_{i}^{P}=$ parâmetro autorregressivo sazonal;

$\ominus_{j}^{Q}=$ parâmetro de média móvel sazonal; e

$\delta=$ constante

De acordo com Gujarati (2006), as etapas para a construção do modelo de Box e Jenkins podem ser divididas em quatro: (1) identificação, (2) estimação, (3) diagnóstico e (4) previsão.

\section{(1) Identificação}

A etapa de identificação consiste em descobrir qual a melhor modelagem para descrever a série temporal, ou seja, envolve a determinação da ordem do modelo necessário para capturar as características dinâmicas dos dados.

Para a identificação de processos ARIMA e SARIMA, recorre-se às funções de autocorrelação (ACF) e de autocorrelação parcial (PACF). A partir destas funções identificam-se os valores de $p$ e $P$ para o processo AR e os valores de $q$ e $Q$ para o processo MA. Portanto, na fase de identificação, tem-se a ACF e a PACF como ferramentas básicas para verificação do comportamento da série e observação dos valores de $p, P, d, D, q, Q$ e $s$.

No entanto, nesta etapa de identificação do modelo, não é mais comum analisar somente os gráficos das funções ACF e PACF, pois dados reais raramente apresentam padrões simples como os mostrados nos livros textos.

Atualmente, os critérios de seleção para modelos ARIMA mais utilizados são o AIC (Akaike information criterion) e o BIC (Bayesian information criterion). Esses critérios incorporam um termo de penalidade para o aumento do número de parâmetros ( $p, P, q$ e $Q$ ) no modelo, de forma que modelos mais parcimoniosos sejam escolhidos. O critério AIC superestima assintoticamente a ordem verdadeira do modelo (Granger e Newbold, 1986) apresentando tendência a escolher modelos superparametrizados. Por outro lado, o AIC é assintoticamente eficiente para modelos puramente autorregressivos. O BIC é um critério consistente, de forma que fornece estimativas que convergem em probabilidade para os valores verdadeiros à medida que $T$ tende ao infinito (Brockwell e Davis, 1996). Por esse motivo, optou-se por utilizar este último critério para a escolha do melhor modelo. A equação para esse critério, de acordo com Enders (2004), pode ser representada da seguinte forma: 


$$
B I C=T \ln (S Q R)+n \ln (T)
$$

onde:

$S Q R$ é a soma dos quadrados dos resíduos;

$n$ é o número dos parâmetros estimados;

$T$ é o número de observações utilizadas. Na prática, deve-se aceitar o modelo que apresenta o BIC de menor valor.

\section{(2) Estimação}

Uma vez identificados os valores de $p, P, d, D, q, Q$ e $s$, passa-se para a estimação dos parâmetros dos termos auto-regressivos e de médias móveis incluídos no modelo, como é mostrado na Equação (2). Neste trabalho, usou-se o pacote estatístico Eviews para esta estimação, que pode ser feita através do método dos mínimos quadrados ou de métodos não lineares.

\section{(3) Diagnóstico}

Nesta etapa verifica-se se o modelo previamente identificado e estimado é o modelo mais adequado. A correta especificação de um modelo ARIMA ou SARIMA é verificada no termo de erro $\left(\mu_{t}\right)$, pois ele deve constituir um processo white noise (Granger e Newbold, 1986). Assim, a verificação da adequabilidade do modelo é efetuada nas autocorrelações amostrais dos erros $\left(\mu_{t}\right)$, as quais seguem assintoticamente uma distribuição normal, com média zero e variância constante se forem provenientes de um ruído branco. Para esta análise utilizou-se a estatística desenvolvida por Ljung-Box (LB), dada pela seguinte expressão:

$$
L B=n(n+2) \sum_{k=1}^{m}\left(\frac{\tau_{k}^{2}}{n-k}\right) \sim \chi_{m}^{2}
$$

\section{(4) Previsão}

Um dos métodos de escolha do melhor mecanismo de previsão é a comparação dos valores previstos $\left(\hat{y}_{t}\right)$ com os valores observados da série $\left(y_{t}\right)$, o que caracteriza capacidade preditiva do modelo utilizado. Pode-se definir os resíduos como a diferença entre os valores observados e os valores previstos.

Utilizou-se o erro percentual absoluto médio (MAPE) que considera o erro relativo de cada previsão. Então,

$$
M A P E=\sum_{t=T+1}^{T+h}\left|\frac{\mu_{t}}{y_{t}}\right| / h
$$

O MAPE tem sido utilizado em diversos trabalhos como em Foster (1977), Bathke Jr. e Lorek (1984), Brown e Rozeff (1979) e Lorek e Willinger (2007). 


\subsection{Modelos propostos}

Os modelos propostos na literatura internacional para a previsão dos lucros trimestrais podem ser descritos através dos modelos de Foster (1977), Griffin (1977), Watts e Leftwich (1977) e Brown e Rozeff (1979). Para o presente trabalho serão testados seis modelos, apresentados a seguir, para descrever as séries de lucros das empresas brasileiras. O cinco primeiros são modelos já predeterminados (premier models) e o sexto é a modelagem feita pelo método Box e Jenkins para cada empresa.

- Modelo (1): Random Walk com deslocamento (drift): (RWD)

$$
y_{t}=\delta+y_{t-1}+\mu_{t}
$$

onde $y_{t-1}=$ lucro trimestral no período $t-1$.

O modelo RWD tem sido usado para prever as séries de lucros tanto anuais quanto trimestrais. Por ser um modelo parcimonioso é um candidato natural a ser analisado.

- Modelo (2): Random Walk com deslocamento sazonal: (SRWD)

$$
y_{t}=\delta+y_{t-4}+\mu_{t}
$$

onde $y_{t-4}=$ lucro trimestral no período $t-4$.

Foi incluído o modelo SRWD por diversas razões. Primeiro, o modelo tem sido empregado extensivamente na literatura pertinente, Bernard e Thomas (1990), Ball e E. (1996) e Lorek e Willinger (2007), entre outros. Segundo, o modelo capta os efeitos sazonais que podem surgir, por exemplo, no último trimestre de cada ano devido ao aumento no consumo dado pelas famílias. Conforme discutido por Bell e Hillmer (1983), quando se encontra um ciclo estocástico em dados trimestrais, por exemplo, sugere-se que os dados apresentam variação de calendário "trading day variation". Terceiro, assim como o modelo RWD este também é parcimonioso por natureza.

- Modelo (3): Foster SARIMA $(100) \times(010)$

$$
y_{t}=\delta+y_{t-4}+\varphi_{1}\left(y_{t-1}-y_{t-5}\right)
$$

onde:

$\varphi_{1}=$ parâmetro autorregressivo;

$y_{t-5}=$ lucro trimestral no período $t-5$.

Foster (1977) identificou um modelo autorregressivo com diferença sazonal como o melhor modelo ARIMA para descrever as séries dos lucros trimestrais. 
- Modelo (4): Brown e Rozeff SARIMA (100) × (011)

$$
y_{t}=y_{t-4}+\varphi_{1}\left(y_{t-1}-y_{t-5}\right)-\Theta_{1} \mu_{t-4}
$$

onde $\varphi_{1}=$ parâmetro de médias móveis sazonal;

$\mu_{t-4}=$ termo de erro no período $t-4$.

A principal diferença entre o modelo proposto por Brown e Rozeff (1979) e Foster (1977) é que o primeiro inclui um termo de erro defasado no período 4, portanto adicionou-se um parâmetro de média móvel no modelo.

- Modelo (5): Griffin-Watts SARIMA $(011) \times(011)$

$$
y_{t}=y_{t-4}+\left(y_{t-1}-y_{t-5}\right)-\Theta_{1} \mu_{t-1}-\Theta_{1} \mu_{t-4}-\theta_{1} \Theta_{1} \mu_{t-5}
$$

- Modelo (6): Box e Jenkins (BJ) Para a estimação deste modelo será utilizada a metodologia de Box e Jenkins através da equação (2). Será verificado a significância dos parâmetros, o critério de BIC, a estatística Q e o MAPE, que indicarão o melhor modelo para cada empresa/setor. Espera-se que os modelos encontrados na referida metodologia possuam os menores valores de BIC. Tal modelo foi incluído para testar se a previsão das séries de lucro líquido e operacional deve ser realizada individualmente para cada empresa usando o método de Box e Jenkins ou se algum dos modelos predeterminados é melhor.

\section{Resultados}

Para a etapa de identificação, utilizaram-se os correlogramas ACF e PACF, posteriormente procedeu-se à significância dos parâmetros, condições de estacionariedade e invertibilidade, menores valores do critério de seleção $B I C$, estatística LB insignificante e menor erro de previsão dado pelo MAPE.

Verificaram-se quatro situações para os correlogramas (ACF e PACF). O primeiro, com a série em nível para a identificação dos modelos 1 e 2 propostos. $\mathrm{O}$ segundo, com a série em primeira diferença. O terceiro, em primeira diferença e primeira diferença sazonal, a fim de identificar o modelo proposto por Griffin (1977) e Watts (1975). E o quarto, com uma diferença sazonal para identificar o modelo proposto por Brown e Rozeff (1979).

\subsection{Modelos propostos e critérios de seleção}

Os resultados abaixo dizem respeito aos modelos ARIMA e SARIMA e aos critérios de escolha dos modelos mais adequados. A Tabela 1 apresenta os resultados médios referentes aos critérios de seleção dos modelos predeterminados para o lucro líquido e lucro operacional. Como salientado anteriormente, utilizou-se o critério BIC (Bayesian information criterion). 
Tabela 1

Critérios de seleção (valores do BIC) dos modelos predeterminados para as séries do lucro líquido e lucro operacional. A média foi utilizada para a realização dos cálculos para o período de 1995 a 2008

\begin{tabular}{|c|c|c|c|c|c|c|}
\hline \multirow[b]{2}{*}{ Setores } & \multicolumn{6}{|c|}{ Modelos } \\
\hline & RWD & SRWD & Foster & $\mathrm{BR}$ & GW & $\overline{\mathrm{BJ}}$ \\
\hline & \multicolumn{6}{|c|}{ Lucro Líquido } \\
\hline Bens Industriais & 18,39 & 18,64 & 18,71 & 18,42 & 18,49 & 18,29 \\
\hline Construção e Transporte & 15,37 & 15,36 & 15,74 & 15,44 & 15,57 & 15,29 \\
\hline Consumo Cíclico & 18,02 & 17,97 & 18,31 & 17,98 & 18,05 & 17,78 \\
\hline Consumo Não Cíclico & 20,76 & 20,84 & 21,15 & 20,75 & 20,86 & 20,62 \\
\hline Materiais Básicos & 20,74 & 21,13 & 21,25 & 20,84 & 20,9 & 20,65 \\
\hline Petróleo, Gás e Biocombustível & 22,37 & 22,81 & 22,94 & 22,54 & 22,58 & 22,19 \\
\hline Tecnologia da Informação & 20,1 & 20,16 & 20,38 & 20,26 & 20,38 & 19,92 \\
\hline Telecomunicação & 23,75 & 23,92 & 24,01 & 23,72 & 23,71 & 23,56 \\
\hline Utilidade Pública & 22,78 & 22,9 & 23,4 & 22,97 & 23,05 & 22,71 \\
\hline \multirow[t]{2}{*}{ Média para os modelos } & 20,25 & 20,41 & 20,65 & 20,32 & 20,4 & 20,11 \\
\hline & \multicolumn{6}{|c|}{ Lucro Operacional } \\
\hline Bens Industriais & 17,69 & 18,15 & 17,93 & 17,62 & 17,69 & 17,46 \\
\hline Construção e Transporte & 15,61 & 15,74 & 15,95 & 15,71 & 15,76 & 15,53 \\
\hline Consumo Cíclico & 16,92 & 16,96 & 16,94 & 16,71 & 16,79 & 16,56 \\
\hline Consumo Não Cíclico & 19,51 & 19,95 & 19,84 & 19,41 & 19,52 & 19,28 \\
\hline Materiais Básicos & 20,11 & 20,96 & 20,54 & 20,09 & 20,14 & 20,03 \\
\hline Petróleo, Gás e Biocombustível & 22,73 & 24,2 & 22,92 & 22,73 & 22,72 & 22,58 \\
\hline Tecnologia da Informação & 18,85 & 19,14 & 19,46 & 19,14 & 19,31 & 18,83 \\
\hline Telecomunicação & 23,13 & 24,2 & 22,92 & 22,73 & 22,72 & 22,58 \\
\hline Utilidade Pública & 22,28 & 22,46 & 22,7 & 22,41 & 22,36 & 22,16 \\
\hline Média para os modelos & 19,65 & 20,2 & 19,91 & 19,62 & 19,67 & 19,45 \\
\hline \multicolumn{7}{|l|}{ Observações: } \\
\hline $\begin{array}{l}\text { RWD = random walk com deslocam } \\
\text { F = Modelo proposto por Foster SAI } \\
\text { Brown e Rozeff SARIMA (100) }(01 \\
\text { GW = Modelo proposto por Griffin } \\
\text { BJ = Melhor modelo selecionado ap }\end{array}$ & $\begin{array}{l}\text { to; SRWI } \\
\text { MA (100) }\end{array}$ & $\begin{array}{l}=\text { random } \\
010) ; \mathrm{BR}=\end{array}$ & $\begin{array}{l}\text { lk com de } \\
\text { Modelo pro }\end{array}$ & $\begin{array}{l}\text { ocament } \\
\text { osto por }\end{array}$ & sazonal; & \\
\hline
\end{tabular}

Observa-se que para todos os setores a metodologia proposta por Box e Jenkins apresenta os menores valores dos critérios de seleção, indicando que as empresas devem ser modeladas individualmente.

A fim de corroborar com os modelos propostos pela literatura internacional pertinente, serão analisados nesta alínea somente os cinco modelos predeterminados. Nota-se que o modelo RWD apresenta os menores valores pelo critério de seleção, sendo assim tal modelo é o que melhor descreve as autocorrelações para a série do lucro líquido.

Para a série do lucro operacional, observa-se que o modelo de BR apresenta conjuntamente o menor valor médio do critério de seleção, excluindo o modelo BJ.

\subsection{Diagnóstico}

Os resultados referentes aos resíduos de acordo com a estatística da função de autocorrelação estão apresentados na Tabela 2. Tal estatística sugere que se o 
modelo estiver bem ajustado aos dados pode-se aceitar a hipótese de que não existe autocorrelação entre os termos de erros estocásticos, segundo Gujarati (2006).

Os resultados médios da estatística Ljung-Box (LB) são apresentados abaixo para a série do lucro líquido e do lucro operacional.

Tabela 2

Diagnóstico (estatística Ljung-Box - LB) dos modelos predeterminados para as séries do lucro líquido e lucro operacional. A média foi utilizada para a realização dos cálculos para o período de 1995 a 2008

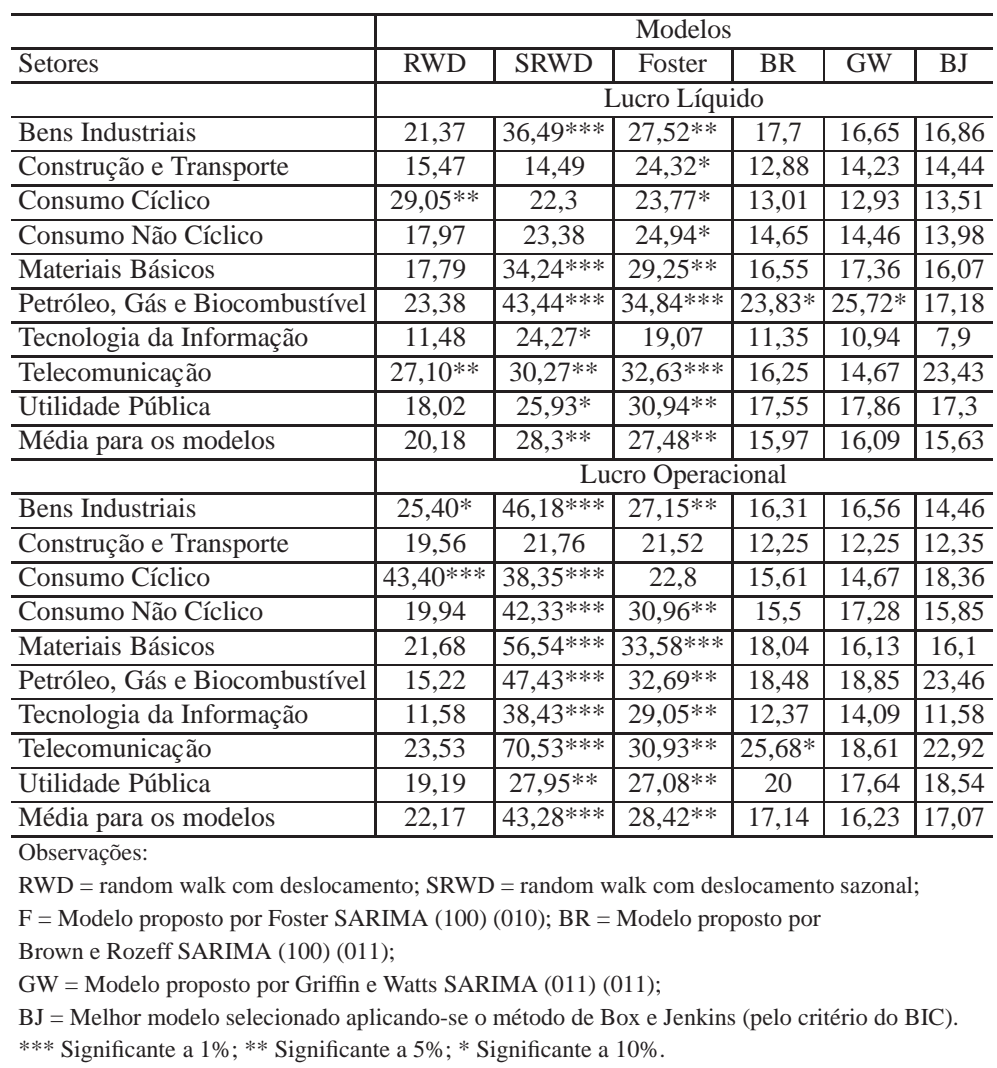

Observa-se, para o lucro líquido, que os modelos SRWD e de Foster apresentam autocorrelações significantes para a maioria dos setores, isto significa que os modelos não se ajustam bem aos dados. Para o modelo $R W D$ observa-se que somente os setores de consumo cíclico e de telecomunicação apresentam-se significantes ao nível de 5\%. Para os modelos BR e GW o setor de petróleo, gás e biocombustível permite rejeitar a hipótese nula de que os resíduos não são autocorrelacionados ao nível de significância de $10 \%$. Para o modelo BJ todos os setores apresentam-se não autocorrelacionados entre os resíduos de acordo com a estatística LB, isto é, tal modelo capta com acuidade todas as autocorrelações existentes entre a série do lucro líquido. 
Os modelos SRWD e Foster parecem não captar com acuidade as oscilações da série do lucro operacional, pois os resultados das estatísticas de LB permitem a rejeição da hipótese de que os resíduos não são correlacionados. Observa-se que os setores de bens industriais e consumo cíclico apresentam correlação entre os resíduos mesmo depois de aplicado o modelo AR (1). O modelo de BR ajusta-se bem aos dados, com exceção do setor de telecomunicação. Os modelos de GW e BJ parecem não apresentar autocorrelação entre os resíduos.

\subsection{Previsão}

Os valores médios dos MAPEs para os modelos selecionados estão expostos na Tabela 3 para a série do lucro líquido e operacional.

Vale ressaltar que os modelos a serem utilizados para a realização da previsão devem ser aqueles que apresentaram o menor valor de BIC conforme o exposto no item 3.2. Portanto, além dos modelos BJ, os modelos RWD e BR devem também ser utilizados para a realização das previsões para a série do lucro líquido e operacional, respectivamente. Vale ressaltar que a Tabela 3 refere-se à média de previsão para os quatro períodos analisados, isto é, foram realizadas previsões para o período do último trimestre de 2008 , tomando como base um passo à frente o trimestre de setembro/2008, dois passos à frente o trimestre de junho/2008, três passos à frente o trimestre de março/2008 e quatro passos à frente o trimestre de dezembro/2007.

Corroborando com os critérios de seleção observa-se que o modelo BJ é o que apresenta o menor erro de previsão para todos os setores selecionados se considerarmos a média para os modelos. O modelo BR é o que realiza as melhores previsões, se excluirmos a metodologia BJ, para a série do lucro operacional confirmando com o exposto anteriormente. Tal fato não é apresentado para a série do lucro líquido para o modelo RWD, pois este apresenta previsões inferiores aos demais modelos.

Ao considerarmos apenas os cinco modelos predeterminados, observa-se que o modelo proposto por Brown e Rozeff (1979) apresenta os menores valores médios dos MAPEs para os períodos utilizados na realização das previsões, tanto para as séries do lucro líquido como operacional.

Nota-se que os valores dos MAPEs para a série do lucro líquido são na média maiores que os obtidos na série do lucro operacional, com exceção do modelo SRWD, isto é, o lucro operacional apresenta em média uma capacidade preditiva melhor que o lucro líquido. Tal fato pode ser atribuído às maiores oscilações a que o lucro líquido está exposto, pois no seu cálculo leva-se em conta os resultados não-operacionais e itens extraordinários das empresas. 
Tabela 3

Valores dos erros de previsão (MAPE) para os modelos predeterminados para as séries do lucro líquido e lucro operacional. A média foi utilizada para a realização dos cálculos para o período de 1995 a 2008

\begin{tabular}{l|c|c|c|c|c|c}
\hline & \multicolumn{5}{|c}{ Modelos } \\
\hline Setores & RWD & SRWD & Foster & BR & GW & BJ \\
\hline & \multicolumn{7}{|c|}{ Lucro Líquido } \\
\hline Bens Industriais & 1,75 & 1,79 & 1,67 & 1,52 & 2,33 & 1,2 \\
\hline Construção e Transporte & 2,86 & 2,94 & 4,01 & 3,58 & 5,66 & 2,66 \\
\hline Consumo Cíclico & 2,85 & 2,66 & 3,18 & 2,4 & 3,08 & 2,07 \\
\hline Consumo Não Cíclico & 3,63 & 4,21 & 4,08 & 3,79 & 4,35 & 3,81 \\
\hline Materiais Básicos & 2,63 & 3,08 & 2,67 & 1,9 & 2,33 & 1,93 \\
\hline Petróleo, Gás e Biocombustível & 1,04 & 1,1 & 0,9 & 0,68 & 0,72 & 0,63 \\
\hline Tecnologia da Informação & 1,61 & 1,63 & 1,84 & 1,96 & 4,16 & 1,06 \\
\hline Telecomunicação & 8,91 & 4,98 & 4,42 & 2,51 & 1,4 & 2,88 \\
\hline Utilidade Pública & 2,68 & 2,23 & 3,34 & 2,29 & 3,75 & 2,23 \\
\hline Média para os modelos & 3,11 & 2,74 & 2,9 & 2,29 & 3,09 & 2,05 \\
\hline & \multicolumn{7}{|c|}{ Lucro Operacional } \\
\hline Bens Industriais & 3,19 & 2,74 & 2,07 & 1,94 & 2,21 & 1,76 \\
\hline Construção e Transporte & 2,99 & 4,2 & 3,93 & 2,44 & 3,22 & 1,97 \\
\hline Consumo Cíclico & 3,79 & 3,18 & 2,65 & 2,47 & 3,19 & 2,14 \\
\hline Consumo Não Cíclico & 1,76 & 1,96 & 2,69 & 1,76 & 2,25 & 1,32 \\
\hline Materiais Básicos & 1,98 & 3,79 & 1,74 & 1,49 & 1,52 & 1,42 \\
\hline Petróleo, Gás e Biocombustível & 1,84 & 3,43 & 1,64 & 0,92 & 0,91 & 0,98 \\
\hline Tecnologia da Informação & 2,12 & 2,35 & 2,44 & 2,44 & 1,82 & 1,53 \\
\hline Telecomunicação & 0,44 & 0,72 & 0,54 & 0,37 & 0,37 & 0,39 \\
\hline Utilidade Pública & 3,16 & 9,14 & 7,38 & 2,53 & 3,6 & 2,76 \\
\hline Média para os modelos & 2,36 & 3,5 & 2,79 & 1,82 & 2,12 & 1,59 \\
\hline Obsvços:
\end{tabular}

Observações:

RWD = random walk com deslocamento; SRWD = random walk com deslocamento sazonal;

$\mathrm{F}=$ Modelo proposto por Foster SARIMA (100) (010); BR = Modelo proposto por

Brown e Rozeff SARIMA (100) (011); GW = Modelo proposto por Griffin e Watts SARIMA (011) (011);

$\mathrm{BJ}=$ Melhor modelo selecionado, pelo critério BIC, aplicando-se o método de Box e Jenkins.

\subsection{Modelos individuais}

Vale ressaltar que os modelos individuais foram aqueles encontrados com a aplicação da metodologia proposta por Box e Jenkins (1976). A fim de simplificar a análise dos resultados, as Tabelas 4 e 5 apresentam os modelos identificados para o lucro líquido e lucro operacional, respectivamente. Tais modelos são importantes para verificar se existe um perfil comum de identificação para as séries de lucro em estudo.

Observa-se que não é possível pela análise das empresas da amostra determinar um único perfil de previsibilidade para todas as empresas e setores para o lucro líquido e operacional. Não existem setores ou modelo (S)ARIMA específicos que determinam características comuns das empresas ou setores. 
No entanto, a partir da análise individual dos modelos identificados para cada empresa observou-se uma concentração nos modelos $(000) \times(000)$ e $(010) \times$ (000) para o lucro líquido e lucro operacional, respectivamente. Portanto, a análise das empresas deve ser realizada individualmente.

Modelos descritos como $(000) \times(000)$ não podem ser modelados estatisticamente, pois sua série é um típico comportamento white noise, ou seja, não existe autocorrelação entre a série, portanto os lucros não podem ser previstos analisando única e exclusivamente seus dados históricos.

A fim de comparar os modelos propostos pela literatura internacional e a concentração dos modelos encontrados nas empresas brasileiras, a Tabela 6 apresenta as médias do critério de seleção BIC para que possa ser feita tal comparação. Portanto, para a série do lucro líquido, o modelo utilizado pode ser descrito por um ARIMA $(000) \times(000)$ e para a série do lucro operacional, o modelo apresentado pode ser representado por um ARIMA $(010) \times(000)$.

Observa-se que o modelo ARIMA $(000) \times(000)$ para o lucro líquido na média apresenta-se superior apenas para o modelo proposto por Foster (1977). O modelo RWD é o que apresenta o menor valor médio de BIC, portanto tal modelo deve ser o escolhido para captar as oscilações da série do lucro líquido, conforme ressaltado anteriormente.

Vale mencionar que para a série do lucro operacional o modelo ARIMA (010) $\times(000)$ ajusta-se melhor aos dados nos setores de material básico, petróleo, gás e biocombustível e telecomunicação se comparados aos modelos propostos pela literatura internacional pertinente. Porém, em média o modelo proposto por Brown e Rozeff (1979) é o que apresenta o menor valor médio do critério de seleção, portanto o modelo deve ser o escolhido para a realização de previsões para a série do lucro operacional. 
Tabela 4

Lucro Líquido - número de modelos estimados por setor

\begin{tabular}{|c|c|c|c|c|c|c|c|c|c|c|c|c|c|c|c|c|c|c|c|c|c|c|c|c|c|c|c|}
\hline & \multicolumn{27}{|c|}{ Modelos encontrados } \\
\hline Setores & $\mathrm{RW}$ & SRWD & \begin{tabular}{|l|l|}
$\mathrm{F}$ & $\mathrm{BR}$ \\
\end{tabular} & $\mathrm{GW}$ & & 785 & $9 \quad 10$ & 11 & \begin{tabular}{|l|l|l|l|l|l|l|l|l}
12 & 1 \\
\end{tabular} & \begin{tabular}{l|l|l}
13 & 14 \\
\end{tabular} & $\begin{array}{ll}14 & 1: \\
\end{array}$ & $5[1$ & \begin{tabular}{l|l}
6 & 17 \\
\end{tabular} & 718 & 19 & 20 & 212 & 22 & 232 & & 252 & \begin{tabular}{l|l}
26 & 2 \\
\end{tabular} & 728 & 329 & 30 & 31 & 32 \\
\hline Bens Industriais & 2 & 2 & & 1 & & 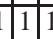 & 1 & 4 & \begin{tabular}{l|l}
1 &
\end{tabular} & \begin{tabular}{l|l}
1 & 3 \\
\end{tabular} & 3 & & & & & & & & & & & & & & & & \\
\hline Construção e Transporte & & & & & & & & & & 3 & \begin{tabular}{l|l}
3 & 1 \\
\end{tabular} & & & & & & & & & & & & & & & & \\
\hline Consumo Cíclico & 5 & 1 & & & & & & 2 & & 8 & 8 & 1 & 12 & 1 & 1 & 1 & 1 & 1 & 1 & 1 & & & & & & & \\
\hline Consumo Não Cíclico & 1 & & & 1 & 1 & 1 & & & & 3 & $\overline{3}$ & & & & & & & & & & \begin{tabular}{l|l}
1 & 1 \\
\end{tabular} & \begin{tabular}{l|l}
1 & 1 \\
\end{tabular} & & & & & \\
\hline Materiais Básicos & 5 & & & & & 1 & & 4 & & 6 & \begin{tabular}{l|l}
6 & 1 \\
\end{tabular} & & 8 & & & & 2 & & & & & & 1 & 1 & 1 & & \\
\hline Petróleo, Gás e Biocombustível & & & & & & 11 & & & & & & & & & & & & & & & 1 & & & & & 1 & \\
\hline Tecnologia da Informação & & & & & & & & & & & & & & & & & & & & & & 1 & & & & & \\
\hline Telecomunicação & & & & & & & & 1 & & 1 & 1 & & & & & & & & & & & & & & & & 1 \\
\hline Utilidade Pública & 3 & & & & & & & 3 & & 4 & 4 & & 1 & & & & & & & & 1 & & & & & & \\
\hline
\end{tabular}

Utilidade Pública

Observações:

Modelos: RW - $(1,0,0)(0,0,0)$; SRWD - $(0,0,0)(1,0,0) ; \mathrm{F}-(1,0,0)(0,1,0) ; \mathrm{BR}-(1,0,0)(0,1,1) ; \mathrm{GW}-(0,1,1)(0,1,1) ; 6-(0,0,1)(0,1,1) ; 7-(0,1,2)(0,0,0) ; 8-(1,1,0)(0,0,0)$;

$9-(0,0,0)(0,1,0) ; 10-(1,0,0)(1,0,1) ; 11-(0,1,1)(0,0,0) ; 12-(1,0,1)(0,0,0) ; 13-(1,1,0)(0,0,1) ; 14-(0,0,0)(0,0,0) ; 15-(0,1,1)(0,0,1) ; 16-(0,1,0)(1,0,0) ; 17-$

$(0,1,0)(0,0,0), 18-(1,1,0)(0,1,0), 19-(0,0,0)(0,1,1), 20-(1,1,2)(0,0,0), 21-(1,0,0)(1,0,0), 22-(0,1,1)(0,1,0), 23-(2,0,0)(2,1,0) ; 24-(2,1,0)(0,0,0) ; 25-(0,0,1)(0,0,0)$;

$26-(1,0,0)(0,0,1) ; 27-(0,0,0)(1,1,0) ; 28-(1,0,0)(1,1,0) ; 29-(0,0,1)(1,1,0) ; 30-(0,1,0)(0,0,1) ; 31-(0,0,1)(0,0,1) ; 32-(0,1,1)(1,0,0)$. 
Tabela 5

Lucro operacional - número de modelos estimados por setor

\begin{tabular}{|c|c|c|c|c|c|c|c|c|c|c|c|c|c|c|c|c|c|c|c|c|c|c|c|c|c|c|c|c|}
\hline & \multicolumn{28}{|c|}{ Modelos encontrados } \\
\hline Setores & $\mathrm{RW}$ & SRWD & & \begin{tabular}{l|l}
$\mathrm{BR}$ & $\mathrm{GW}$ \\
\end{tabular} & \begin{tabular}{l|l|l|l|l}
$N$ & 6 & 7 \\
\end{tabular} & 8 & \begin{tabular}{l|l|}
9 & 10
\end{tabular} & 11 & 12 & 13 & & 15 & 16 & 17 & 18 & & 20 & & 22 & \begin{tabular}{l|l}
23 & 2 \\
\end{tabular} & & 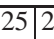 & 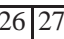 & 728 & 29 & 30 & 31 & 32 \\
\hline Bens Industriais & 2 & & 1 & 1 & 2 & & 1 & & 3 & & 1 & & & & 1 & & & \begin{tabular}{|l|l|l}
1 & $r$
\end{tabular} & 2 & & 1 & \begin{tabular}{l|l}
2 & 1 \\
1
\end{tabular} & 1 & & & & & \\
\hline Construção e Transporte & & 1 & & & & & & & 1 & & 1 & & & & & & & & & & & & 1 & & & & & \\
\hline Consumo Cíclico & 3 & & & 1 & & 1 & 1 & & 1 & 1 & 3 & 1 & 2 & 2 & & 1 & & & 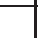 & & & 1 & 3 & 2 & 1 & 1 & & \\
\hline Consumo Não Cíclico & 3 & & & & 1 & & 1 & & 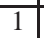 & & 1 & & & & & 1 & & & 1 & & & 1 & & & & & & \\
\hline Materiais Básicos & 2 & & & 2 & $1 / 3$ & & 2 & 1 & & & 8 & & & 2 & 2 & & \begin{tabular}{|l|l}
1 & $r a r a$
\end{tabular} & & & \begin{tabular}{|lllllll}
1 & & $l$ & $l$ & \\
\end{tabular} & & 2 & & 1 & & & 2 & \\
\hline Petróleo, Gás e Biocombustível & & & & & & & 1 & & 1 & & 1 & & & & & & & & & & & 1 & & & & & & \\
\hline Tecnologia da Informação & 1 & & & & & & & & & & & & & & & & & & & & & & & & & & & \\
\hline Telecomunicação & & & & & 1 & 1 & & & & 1 & & & & & & & & & & & & 1 & & & & & & \\
\hline Utilidade Pública & & & & & 1 & 1 & 5 & & 3 & & & & & 1 & & & & 1 & & & & & & & & & & 1 \\
\hline
\end{tabular}

Modelos: RW - $(1,0,0)(0,0,0) ; \mathrm{SRWD}-(0,0,0)(1,0,0) ; \mathrm{F}-(1,0,0)(0,1,0) ; \mathrm{BR}-(1,0,0)(0,1,1) ; \mathrm{GW}-(0,1,1)(0,1,1) ; 6-(0,0,1)(0,1,1) ; 7-(1,1,0)(0,0,0) ; 8-(0,0,0)(0,1,0)$; $9-(1,0,0)(1,0,1) ; 10-(0,1,1)$ $(1,1,2)(0,0,0) ; 18-(2,1,0)(0,0,0), 19-(0,0,1)(0,0,0) ; 20-(1,0,0)(0,0,1), 21-(0,0,1)(1,1,0) ; 22-(0,1,0)(0,0,1) ; 23-(0,0,1)(0,0,1) ; 24-(0,1,3)(0,0,0) ; 25-(0,1,0)(0,1,1)$ $26-(0,1,1)(1,0,1) ; 27-(0,0,0)(0,1,1) ; 28-(0,1,0)(0,1,0) ; 29-(0,0,2)(0,0,0) ; 30-(0,0,0)(0,0,1) ; 31-(0,1,0)(1,1,0) ; 32-(2,0,0)(0,0,0)$. 
Tabela 6

Valores da estatística BIC usada como critério de seleção dos modelos predeterminados para as séries do lucro líquido e lucro operacional. A média foi utilizada para a realização dos cálculos para o período de 1995 a 2008

\begin{tabular}{|c|c|c|c|c|c|c|}
\hline & \multicolumn{6}{|c|}{ Modelos } \\
\hline Setores & RWD & SRWD & Foster & $\mathrm{BR}$ & GW & BJ \\
\hline \multicolumn{7}{|c|}{ Lucro Líquido } \\
\hline Bens Industriais & 18,39 & 18,64 & 18,71 & 18,42 & 18,49 & 18,87 \\
\hline Construção e Transporte & 15,37 & 15,36 & 15,74 & 15,44 & 15,57 & 15,33 \\
\hline Consumo Cíclico & 18,02 & 17,97 & 18,31 & 17,98 & 18,05 & 18,09 \\
\hline Consumo Não Cíclico & 20,76 & 20,84 & 21,15 & 20,75 & 20,86 & 20,09 \\
\hline Materiais Básicos & 20,74 & 21,13 & 21,25 & 20,84 & 20,9 & 21,29 \\
\hline Petróleo, Gás e Biocombustível & 22,37 & 22,81 & 22,94 & 22,54 & 22,58 & 23,04 \\
\hline Tecnologia da Informação & 20,1 & 20,16 & 20,38 & 20,26 & 20,38 & 20,23 \\
\hline Telecomunicação & 23,75 & 23,92 & 24,01 & 23,72 & 23,71 & 24,23 \\
\hline Utilidade Pública & 22,78 & 22,9 & 23,4 & 22,97 & 23,05 & 22,89 \\
\hline Média para os modelos & 20,25 & 20,41 & 20,65 & 20,32 & 20,4 & 20,45 \\
\hline \multicolumn{7}{|c|}{ Lucro Operacional } \\
\hline Bens Industriais & 17,69 & 18,15 & 17,93 & 17,62 & 17,69 & 17,72 \\
\hline Construção e Transporte & 15,61 & 15,74 & 15,95 & 15,71 & 15,76 & 15,85 \\
\hline Consumo Cíclico & 16,92 & 16,96 & 16,94 & 16,71 & 16,79 & 17,17 \\
\hline Consumo Não Cíclico & 19,51 & 19,95 & 19,84 & 19,41 & 19,52 & 19,55 \\
\hline Materiais Básicos & 20,11 & 20,96 & 20,54 & 20,09 & 20,14 & 20,03 \\
\hline Petróleo, Gás e Biocombustível & 22,73 & 24,2 & 22,92 & 22,73 & 22,72 & 22,58 \\
\hline Tecnologia da Informação & 18,85 & 19,14 & 19,46 & 19,14 & 19,31 & 19,04 \\
\hline Telecomunicação & 23,13 & 24,2 & 22,92 & 22,73 & 22,72 & 22,7 \\
\hline Utilidade Pública & 22,28 & 22,46 & 22,7 & 22,41 & 22,36 & 22,48 \\
\hline Média para os modelos & 19,65 & 20,2 & 19,91 & 19,62 & 19,67 & 19,68 \\
\hline
\end{tabular}

Observações:

RWD = random walk com deslocamento; SRWD = random walk com deslocamento sazonal;
$F=$ Modelo proposto por Foster SARIMA (100) (010); BR = Modelo proposto por Brown e Rozeff

SARIMA (100) (011); GW = Modelo proposto por Griffin e Watts SARIMA (011) (011);

$\mathrm{BJ}=$ Concentração dos modelos encontrados de acordo com a metodologia Box e Jenkins,

para o lucro líquido (000) x (000) e para o lucro operacional (010) x (000).

\section{Conclusões}

Este artigo analisa o comportamento das séries temporais de lucros trimestrais (operacional e líquido) de empresas brasileiras com ações negociadas em bolsa durante o período de 1995 a 2008. De todo o universo de empresas com ações negociadas na BM\&FBovespa e presentes no banco de dados da empresa Economática, durante o período analisado, 109 obedeceram os critérios de seleção adotados.

Para efetuar a pesquisa, foram utilizados cinco modelos de previsão de séries de lucros já previamente estimados e consagrados na literatura internacional, denominados aqui de modelos predeterminados, bem como foi empregado o método de Box e Jenkins (BJ) para verificar a possibilidade de se encontrar um modelo mais adequado às condições do mercado brasileiro. 
Quanto às séries de lucro operacional, verificou-se, através do método de BJ, que a maioria das empresas parece não ter um comportamento aleatório (random walk), concentrando-se no modelo descrito por um ARIMA $(010) \times(000)$ e ARIMA $(100) \times(011)$. Sendo que este último corrobora o modelo predeterminado de Brown e Rozeff (1979).

Já para a série do lucro líquido, usando o método de BJ, 28 empresas parecem não poder ser modeladas estatisticamente, pois engendrarão um modelo ARIMA $(000) \times(000)$, e 16 empresas parecem ter um comportamento do tipo random walk com drift (RWD), corroborando em parte com a hipótese de que os lucros são aleatórios.

Ao se analisar a média dos critérios de seleção para as séries dos lucros constata-se que a metodologia de Box e Jenkins (1976) deve ser a escolhida se comparada com os cinco modelos propostos pela literatura internacional pertinente.

Ao excluirmos o método de BJ da análise e compararmos os cinco modelos predeterminados entre si, observa-se que o modelo de Brown e Rozeff (1979) e RWD parecem descrever melhor as séries de lucro operacional e lucro líquido, respectivamente.

De um ponto de vista gerencial, os resultados devem ser levados em conta pela administração da empresa em diferentes aspectos. O primeiro refere-se a que o analista não deve usar um modelo predeterminado para suas previsões de lucro, seja operacional ou líquido. Para uma previsão mais precisa o ideal seria estimar um modelo para cada empresa usando o método de BJ, hoje disponível em muitos aplicativos estatísticos. Outro aspecto relaciona-se às previsões usando-se séries de lucro líquido passadas. Estas previsões podem se tornar mera especulação, já que na maioria das empresas aqui analisadas o lucro líquido é descrito por um modelo random walk, ou seja, a melhor previsão para o lucro líquido trimestral seria descrita pelo seu valor no período anterior mais um choque aleatório. Quanto ao lucro operacional, constatou-se que é passível de previsão e o modelo proposto por Brown e Rozeff (1979) pode ser usado, como aproximação, em boa parte das empresas da amostra. A diferença encontrada na modelagem entre o lucro operacional e lucro líquido deve-se ao fato de que no cálculo deste último leva-se em conta os resultados não-operacionais e itens extraordinários das empresas, o que pode tornar mais volátil esta série temporal.

\section{Referências}

Albrecht, W. S., Lookabill, L. L., \& McKeown, J. C. (1977). The time-series properties of annual earnings. Journal of Accounting Research, 15:226-244.

Ariff, M. \& Aw, K. (1987). Lagged variable model applied to dividend behavior in the Singapore equity market: 1973-1986. Proceedings of the Accounting Association of Australia and New Zealand, Auckland, New Zealand.

Austin, L. \& Graydon, D. (1993). Time series properties of company earnings 
and cash flows: Some NZ empirical evidence. Accounting Research Journal, 28:15-24.

Ball, R. \& Brown, P. (1968). An empirical evaluation of accounting income numbers. Journal of Accounting Research, 6:159-178.

Ball, R. \& E., B. (1996). How naive is the stock market's use of earnings information? Journal of Accounting and Economics, 21:319-337.

Ball, R. \& Watts, R. L. (1972). Some time series properties of accounting income. Journal of Finance, 27:663-681.

Ball, R. \& Watts, R. L. (1979). Some additional evidence on survival biases. Journal of Finance, 34:197-206.

Bao, B. H. \& Bao, D. H. (1996). The time series behavior and predictive-ability results of annual value added data. Journal of Business Finance \& Accounting, 23:449-460.

Bathke Jr., A. W. \& Lorek, K. S. (1984). The relationship between time-series models and the security market's expectation of quarterly earnings. Accounting Review, 59:163-176.

Beaver, W. H. (1970). The time series behavior of earnings. Journal of Accounting Research, 8:62-99.

Beaver, W. H., Lambert, R. A., \& Morse, D. (1980). The information content of security prices. Journal of Accounting and Economics, 2:3-28.

Beaver, W. H., Lambert, R. A., \& Ryan, S. G. (1987). The information content of security prices: A second look. Journal of Accounting and Economics, 9:139157.

Beaver, W. H. \& Morse, D. (1978). What determines price-earnings ratios? Financial Analysts Journal, 34:65-76.

Bell, W. R. \& Hillmer, S. C. (1983). Modeling time series with calendar variation. Journal of the American Statistical Association, 78:526-534.

Benston, G. J. \& Watts, R. L. (1978). The market's forecast of earnings. Working Paper, University of Rochester.

Bernard, V. L. \& Thomas, J. K. (1990). Evidence that stock prices do not fully reflect the implications of current earnings for future earnings. Journal of Accounting and Economics, 13:305-340.

Box, G. E. P. \& Jenkins, G. M. (1976). Times Series Analysis: Forecasting and Control. Holden-Day, San Francisco, 2nd edition. 
Brockwell, P. J. \& Davis, R. A. (1996). Introduction to Time Series and Forecasting. Springer-Verlag, New York.

Brooks, L. D. \& Buckmaster, D. A. (1976). Further evidence on the time series properties of accounting income. Journal of Finance, 31:1359-1373.

Brown, L. D., Richardson, G. D., \& Schwager, S. J. (1987). An information interpretation of financial analyst superiority in forecasting earnings. Journal of Accounting Research, 25:49-67.

Brown, L. D. \& Rozeff, M. S. (1978). The superiority of analyst forecasts as a measure of expectations: Evidence from earnings. Journal of Finance, 33:116.

Brown, L. D. \& Rozeff, M. S. (1979). Univariate time-series models of quarterly accounting earnings per share: A proposed model. Journal of Accounting Research, 17:179-189.

Brown, P. \& Neiderhoffer, V. (1968). The predictive content of quarterly earnings. Journal of Business, 41:488-497.

Caird, K. G. \& Emanuel, D. M. (1981). Some time series properties of accounting income numbers. Australian Journal of Management, 6:7-16.

Capstaff, J., Paudyal, K., \& Rees, W. (1998). Earnings forecasting and the earnings to price relationship. Working Paper, Glasgow University, Department of Accounting and Finance.

Chan, L. K. C., Karceski, J., \& Lakonishok, J. (2003). The level and persistence of growth rates. Journal of Finance, 58:643-684.

Collins, D. W. \& Kothari, S. P. (1989). An analysis of intertemporal and crosssectional determinants of earnings response coefficients. Journal of Accounting and Economics, 11:143-181.

Collins, W. A. \& Hopwood, W. S. (1980). A multivariate analysis of annual earnings forecasts generated from quarterly forecast of financial analysts and univariate time series models. Journal of Accounting Research, 18:390-406.

Cunha, F. C. G. (2004). A não-persistência e a variabilidade das taxas de crescimento de empresas de capital aberto listadas na BOVESPA no período 19942002. Dissertação de Mestrado, PUC/Rio, Departamento de Administração.

Dechow, P. M. \& Dichev, I. D. (2002). The quality of accruals and earnings: The role of accrual estimation errors. Accounting Review, 77:35-59.

Enders, W. (2004). Applied Econometric Times Series. Wiley, New Jersey, 2nd edition. 
Fama, E. \& French, K. (2000). Forecasting profitability and earnings. Journal of Business, 73:161-175.

Fama, E. \& French, K. (2002). The equity premium. Journal of Finance, 57:637659.

Finn, F. J. \& Whittred, G. P. (2007). On the use of naïve expectations of earnings per share as experimental benchmarks. Economic Records, 58:169-173.

Firth, M. A. (1982). Some time series properties of corporate earnings in New Zealand: A note. Journal of Business Finance \& Accounting, 9:353-359.

Foster, G. (1977). Quarterly accounting data: Time-series properties and predictive ability results. Accounting Review, 52:1-21.

Foster, G. (1986). Financial Statement Analysis. Prentice Hall, Englewood Cliffs, 2nd edition.

Freeman, R. N., Ohlson, J. A., \& Penman, S. H. (1982). Book rate-of-return and prediction of earnings changes: An empirical investigation. Journal of Accounting Research, 20:639-653.

Granger, C. W. J. \& Newbold, P. (1986). Forecasting Economic Time Series. Academic Press, New York.

Griffin, P. A. (1977). The time-series behavior of quarterly earnings: Preliminary evidence. Journal of Accounting Research, 15:71-83.

Gujarati, D. (2006). Econometria Básica. Elsevier/Campus, Rio de Janeiro.

Hamman, W. D., Jordaan, A. C., \& Smit, E. V. D. (2002). Earnings changes: A random walk? Same south african evidence. Investment Analyst's Journal, 41:5-15.

Harris, R. S. \& Marston, F. C. (2001). The market risk premium: Expectational estimates using analysts' forecasts. Journal of Applied Finance, 11.

Kendall, S. \& Zarowin, P. (1990). Time series properties of annual earnings, earnings persistence and earnings response coefficients. Working Paper, New York University.

Khorana, A., Moyer, R., \& Patel, A. (1999). The ex ante risk premium: More pieces of the puzzle. Working Paper, Georgia Institute of Technology.

Kinnunen, J. (1988). The time series properties of accrual versus cash-based income variables: Empirical evidence from listed finish firms. Ph.D. Dissertation, Helsinki School of Economics. 
Kinnunen, J. (1991). The importance of accrual accounting as a determinant of submartingale behavior in annual income numbers: Empirical evidence from Finland. Journal of Business Finance \& Accounting, 18:861-883.

Kothari, S. P. (2001). Capital markets research in accounting. Journal of Accounting and Economics, 31:105-231.

Little, D. (1962). Higgledy piggledy growth. Bulletin of the Oxford University Institute of Economics and Statistics, 4:387-412.

Little, D. \& Rayner, A. (1966). Higgledy piggledy growth again: An investigation of the predictability of company earnings and dividends in the United Kingdom. Bulletin of the Oxford University Institute of Economics and Statistics.

Lobo, G. J., Kwon, S. S., \& Ndubizu, G. A. (1998). The impact of SFAS no. 14 segment information on price variability and earnings forecast accuracy. Journal of Business Finance \& Accounting, 25:969-985.

Lorek, K. S. (1979). Predicting annual net earnings with quarterly earnings timeseries models. Journal of Accounting Research, 17:190-204.

Lorek, K. S. \& Willinger, G. L. (2007). The contextual nature of the predictive power of statistically-based quarterly earnings models. Review of Quantitative Finance and Accounting, 28:1-22.

Lorek, K. S. \& Willinger, G. L. (2008). Time series properties and predictive ability of quarterly cash flows. Advances in Accounting, 24:65-71.

Martinez, A. L., Da Costa Jr., N. C. A., Cupertino, C. M., \& Coelho, R. A. (2008). Propriedades das séries temporais de lucros trimestrais de empresas brasileiras. Revista de Contabilidade e Organizações, 2:19-36.

Ness Jr., W. L. (1995). A internacionalização do mercado de capitais e a capacidade previsionária de analistas de investimento. Anais do $19^{\circ}$ Encontro da Anpad, João Pessoa, 1(2):131-140.

Ramakrishnan, R. \& Thomas, J. (1992). What matters from the past: Market value, book value, or earnings? Earnings valuation and sufficient statistics for prior information. Journal of Accounting, Auditing and Finance, 7:423-464.

Reilly, F. K., Morgenson, D. L., \& West, M. (1972). The predictive ability of alternative parts of interim financial statements. Journal of Accounting Research, 10:105-124.

Salamon, G. L. \& Smith, E. D. (1977). Additional evidence on the time series properties of reported earnings per share: Comment. Journal of Finance, 32:1795-1801. 
Silva, G. S. F. (2000). Auto-projeção de desempenho para empresas de capital aberto do Brasil. Dissertação de Mestrado, PUC/Rio, Departamento de Administração.

Silva, H. R. L. F. D. (1998). A capacidade previsionária no mercado acionário brasileiro: Um estudo focado nas previsões dos analistas de investimentos. Anais do $22^{\circ}$ Encontro da Anpad, Foz do Iguaçu. (CD-ROM).

Taylor, S. L. \& Tress, R. B. (1988). The time series properties of australian accounting data. Accounting and Finance, 28:17-28.

Watts, R. L. (1975). The time-series behavior of quarterly earnings. Manuscript, University of New Castle.

Watts, R. L. \& Leftwich, R. W. (1977). The time series properties of annual accounting earnings. Journal of Accounting Research, 15:253-271.

Watts, R. L. \& Zimmerman, J. L. (1986). Positive Accounting Theory. Prentice Hall, Englewood Cliffs.

Whittred, G. P. (1978). The time series behaviour of corporate earnings. Australian Journal of Management, 3:195-202.

Wu, C., Kao, C., \& Lee, F. (1996). Time series properties of financial series and implications for modeling. Journal of Accounting, Auditing and Finance, 11:277-303. 